\title{
Blister Test as Method of Measuring Adhesion of Solids on a Flat Surface
}

\author{
K. B. Liland ${ }^{1}$, H. Faremo ${ }^{1} \&$ K. M. Furuheim ${ }^{2}$ \\ ${ }^{1}$ SINTEF Energy Research, Postbox 4761 Torgarden, NO-7465 Trondheim, Norway \\ ${ }^{2}$ Nexans Norway \\ Knut.B.Liland@sintef.no
}

\begin{abstract}
The adhesion between coating and a flat metal surface has been investigated by measurements. The setup is based on the formation of a blister by injecting nitrogen gas under pressure between the coating and the substrate. The adhesion energy is determined by recording the pressure as a function of the recorded blister radius development. A laser is moved over the blister in 2D with two programmable step engines to measure the blister profile and thereby the radius of the blister between successive pressure increases. The design and operation of the instrument are described and an evaluation of its performance and limitations is given. The method could be useful for accurate measurement of adhesion of many types of surface coatings to metal and, possibly, to other substrates, as well as for studies of the various factors that influence adhesion. A better accuracy of the laser for measuring the blister profile would improve the setup.
\end{abstract}

We treated aluminium surfaces in different manners (sand paper, polishing and blowing using glass particles) but found no significant impact on the adhesion energy between the x-linked coating layer and the metal. The adhesion energy measurements however have a large scatter. The coating layer that was not $\mathrm{x}$ linked had a much lower adhesion energy.

\section{Introduction}

Most of the work on constrained blisters has examined the de-bonding of the blister from the surface underneath as the pressure is applied from below (Figure 1).

There are several methods to measure adhesion fracture energy [1-6] $G_{a}$. When using the blister method this depends on how the loading pressure is applied, Williams expression from 1969 [7]).

$$
G_{a}=D \frac{a^{4}}{h^{3}} \cdot P_{c r}
$$

The coating layer is lifted by the applied pressure and form a blister [8]. The blister radius stays fixed up to a critical pressure $P_{c r} . D$ is a material constant [7] for the polymer coating or layer.

The basis of the blister test was originally introduced [9] in 1961 as a means of measuring the adhesion between solids and was developed by many authors thereafter. In its simplest form, the blister geometry consists of a coating against a rigid flat substrate

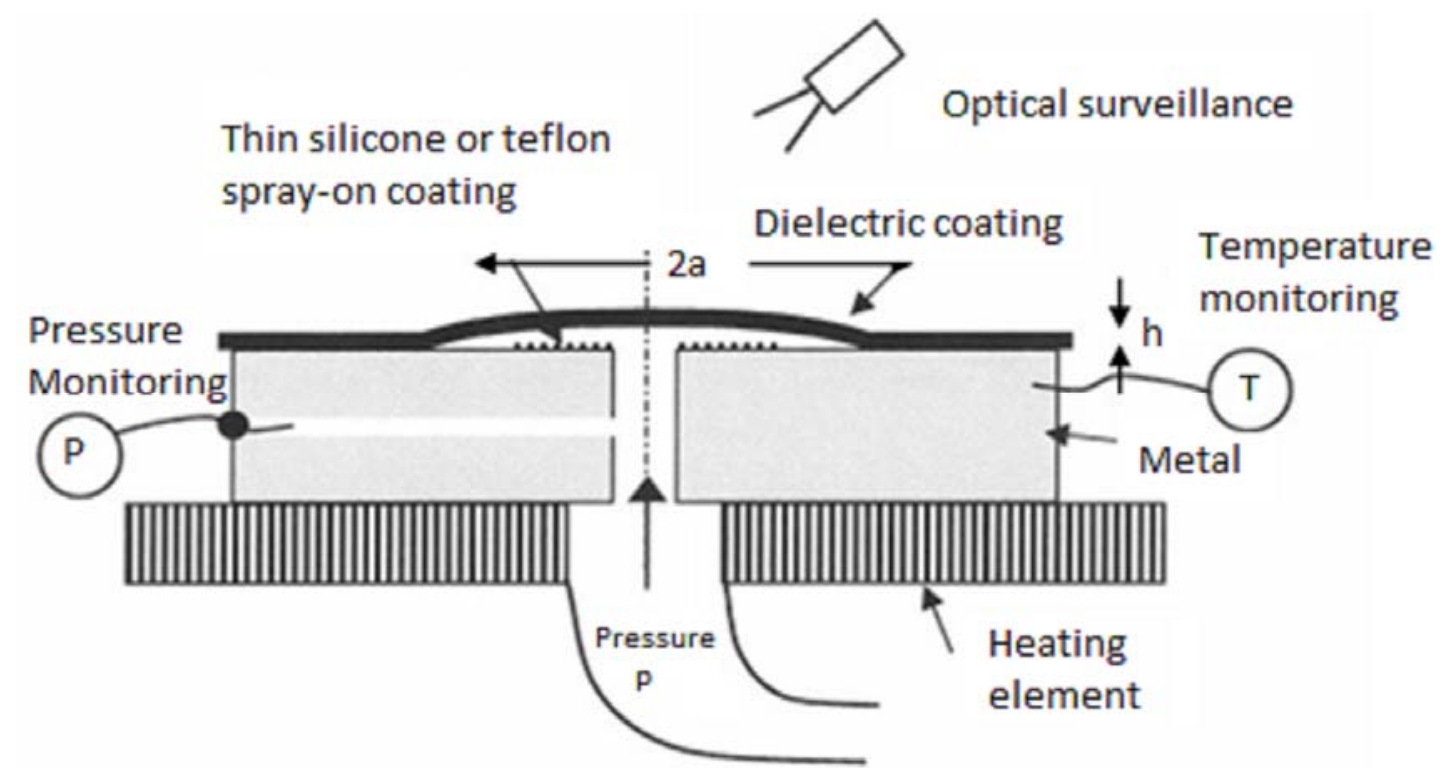

Figure 1: Schematic of the experimental setup for blister test. 
The substrate has a hole (with an unbounded region around the hole initially prepared using Teflon spray-on) in the center. When the unbounded region is progressively pressurized by the pressurizing medium (with gas or a liquid in some cases), the coating forms a blister if it is relatively thin compared with the hole radius. The adhesion energy then can be calculated from the geometry of the blister and the pressure [5]. Any de-bond radius can be used in the test and in principle we can get many measurement points for each specimen if the debonding develops in steps related to corresponding pressure steps. The scan of the blister therefore needs to be fast to be able to detect the development of the blister.

The characterization of surface and interfacial energies between materials is of interest to manufacturer, industry applying the manufactured item, and the scientific community. Eventually the consumers would be affected. The understanding of friction, wear, lubrification, mechanics, adhesive bonding, coating and cleaning of materials would here be an issue. Adhesion is defined as the molecular attraction between the surfaces of bodies in contact. The surface energies of solids are diffucult to measure because their large stiffnesses can often prevent the material from measurably changing shape when adhesive forces are present, that means the material applied to a metal surface might crack before the adhesive energy can be measured (i.e. high temperature cured epoxy becomes very stiff).

In this article we will give a background for the study of the classical blister test and show some results

The focus will be on the classical blister test described in Figure 1. It is possible to separate the blister test into three experimental situations explained by Gent and Lewandowski [10] which is descriptive regarding the blister theory.

1. The blister diameter $2 \mathrm{a}<<$ than the thickness $\mathrm{h}$ of the coating layer.

$$
P_{c r}=\left(\frac{2 \pi E G_{a}}{3 a}\right)^{1 / 2}
$$

giving an adhesion energy:

$$
G_{a}=\frac{P^{2} 3 a}{2 \pi E}
$$

2. The blister diameter $2 \mathrm{a}$ is approximately of the same size as the thickness $h$ of the coating layer.

$$
P_{c r}=\frac{\left(128 E G_{a}\right)^{1 / 2}}{3 a^{2}} h^{3 / 2}
$$

giving an adhesion energy:

$$
G_{a}=\frac{9 a^{4} P^{2}}{128 E h^{3}}
$$

3. The blister diameter $2 \mathrm{a}>>$ than the thickness $\mathrm{h}$ of the coating layer.

$$
P_{c r}={\frac{(17.4 E h)^{1 / 4}}{a}}^{3} G_{a}^{3 / 4}
$$

giving an adhesion energy:

$$
G_{a}=\frac{(P a)^{\frac{4}{3}}}{(17.4 E h)^{\frac{1}{3}}}
$$

The classification into the three modes are connected to how the applied pressure will affect the substrate. The mode where the applied pressure affects the edge of the debonding the most and not the compression and stretching of the substrate material is preferable. If $\mathrm{h}$ becomes large compared to the blister diameter it would be impossible to monitor the blister diameter development.

For the last expression (in 3) we observe that the critical pressure $\left(P_{c r}\right)$ for debonding is less dependent of the tensile modulus $(E)$ and the thickness of the layer $(h)$ and more strongly dependent of the adhesion energy than the other expressions for the other experimental situations (in 1 and 2).

With an elastic behaviour of the coating layer in response to an internal pressure a pressurised blister test could be a good method for measuring interfacial adhesion [10, 11]. Newer literature reviews [12] and [13] gives a good background and compares the different approaches depending on the applications. For the cases 1. and 2. the applied pressure is compressing the material and it will in addition be difficult to observe the curvature of the blister (like a pea under a mattress). When the thickness $\mathrm{h}$ of the coating is thin it is simpler to estimate the debonding diameter.

\section{Experimental setup}

The semiconductor is moulded and $\mathrm{x}$-linked on top the aluminium disc surface described in Figure 2.

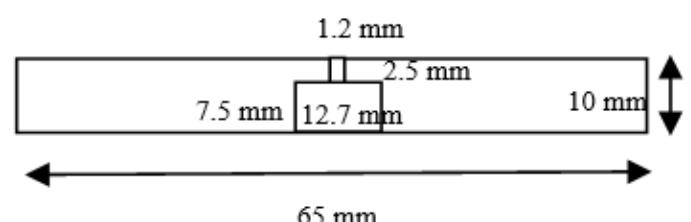

Figure 2: Disc used for the coating of the semi-conductor on the top, the numbers are related to the diameter.

Outside the hole $(1.2 \mathrm{~mm})$ there is a slip zone $(2.5 \mathrm{~mm})$ where Teflon spay is applied in the slip zone. This is done before moulding the coating. A cover plate (Figure 3) with a hole for the spray is applied. The blue area is made of rubber material to avoid the Teflon spray to enter outside the slip zone. The slip zone is used to provide an initial defined area where the coating is not bounded to the aluminium surface. The small $1.2 \mathrm{~mm}$ hole is carefully blocked out using a defined plug to lift the coating outside the slip zone and during moulding of the coating the hole is blocked by a similar but slightly smaller plug. 


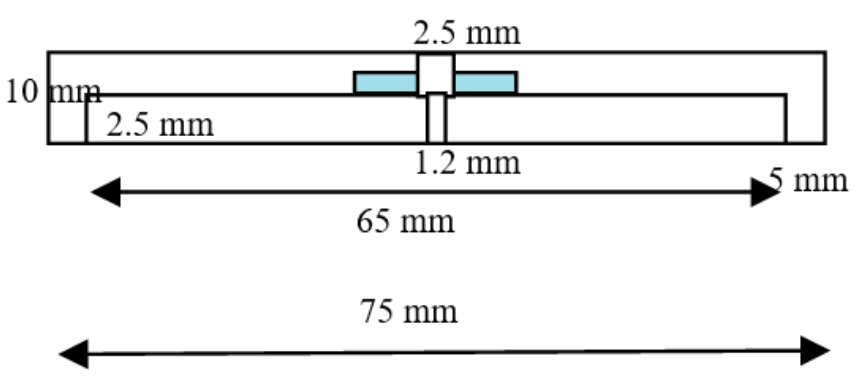

Figure 3: Cover for applying the Teflon spray in the slip zone of the aluminium disc.

The schematic of the setup is shown in Figure 1. In Figure 4 we observe the coating layer (with white paint on top of the coating for reflection purposes) on top of the aluminium disc. Under operation a support ring pressing the disc downwards has been introduced to compensate for the gas pressure coming from below pushing the disc upwards. A non-supported disc would introduce a movement upwards in this setup and make the position of the disc unstable affecting the laser measurements. There will however be a slight drift upward due to the soft coating material being penetrated by the support ring when the gas pressure increases but this can be compensated for using the area outside the small blister zone as "baseline".

\section{Experiment}

The engines are controlled by a XILab script controlling the scanning area grid, engine speed and acceleration between the grid points.

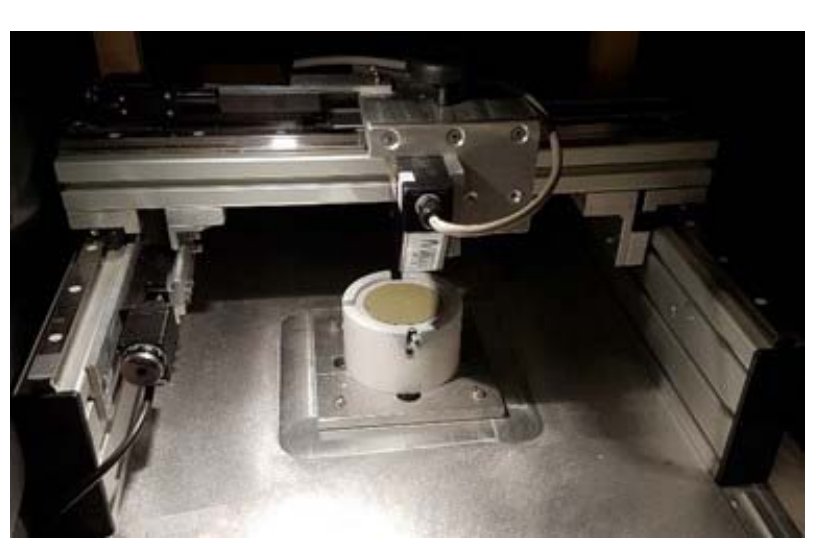

Figure 4: Blister setup with coated and painted disc, engines for moving laser.

\subsection{Aluminium surface treatment}

Three techniques for treating the aluminium disc surfaces were used. 1. Sand paper of different gradings treating the aluminium surface $( \pm 5-10 \mu \mathrm{m}$ ripple variation) in Figure 5 where the blister hole also is observed (black area) in addition to the scratches in the aluminium surface. In Figure 5 (below) we observe the variation in $\mu \mathrm{m}$ along two axis (X-axis and $\mathrm{Y}$-axis) taken from Figure 5 upper picture. This illustrates that the surface variation is different in the two directions due to the one directional sandpaper treatment 2. Another surface treatment is using glass blown particles of $0.25-0.40 \mathrm{~mm}$ size on the aluminium disc giving a more random directional surface variation (also $\pm 5-10 \mu \mathrm{m}$ ) and 3. Polishing the surface smoothly giving a smoother surface variation $( \pm 0.5 \mu \mathrm{m})$.
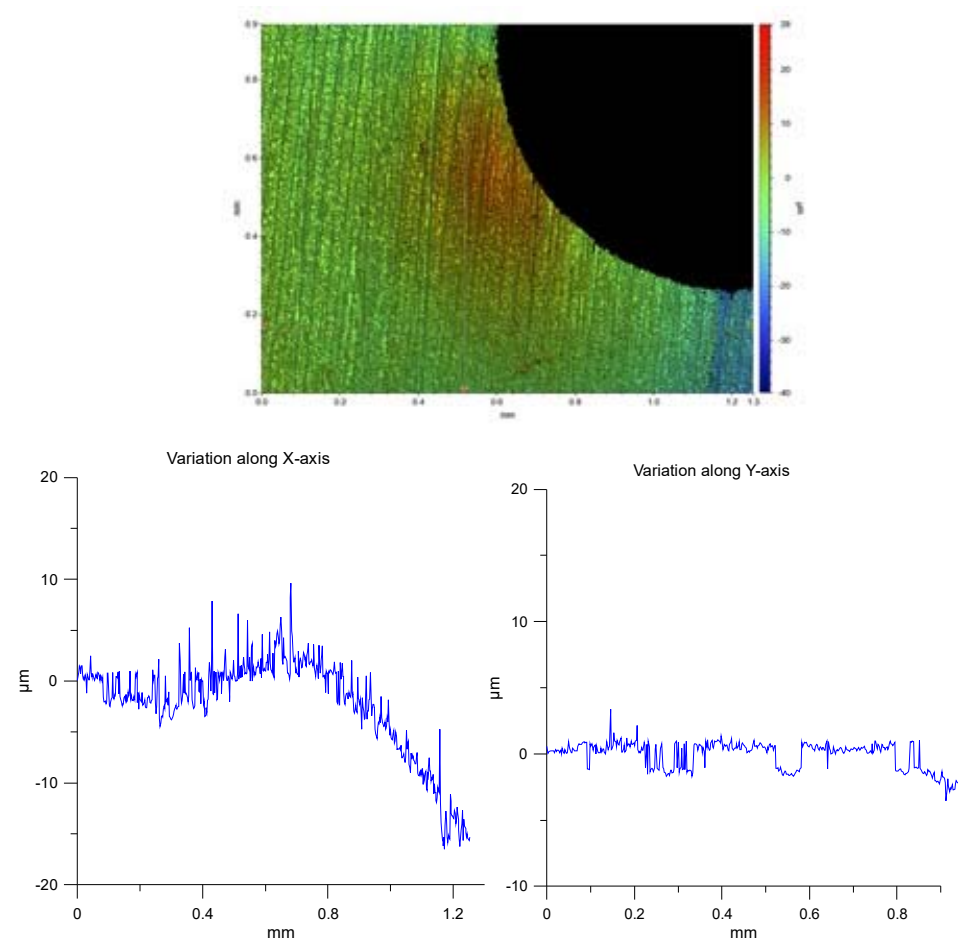

Figure 5: Sand paper treatment (FEPA 320) of aluminium disc surface 


\subsection{One axis measurement}

During the blister profile measurements we first moved the laser along one axis in a forward movement and thereafter in the opposite direction observed in Figure 6. This gives information that the blister has a dynamic movement (mostly in height) after each pressure rise. For the first pressure rise a drop in the pressure is observed between the forward and return movement due to the initial formation and stretching of the material forming the blister. For the higher pressures the pressure remains stable (between forward and return movement) consistent with a very small blister volume increase. To calculate the adhesion energy, we use the pressure observed when the edge of the blister starts to propagate which is an estimate of the critical pressure and the radius a of the blister before the edge moves. The thickness of the coating layer is measured outside the deformed area at three places after the coating layer has been blown off for the whole disc. The measurements also illustrate that a quick scan of the

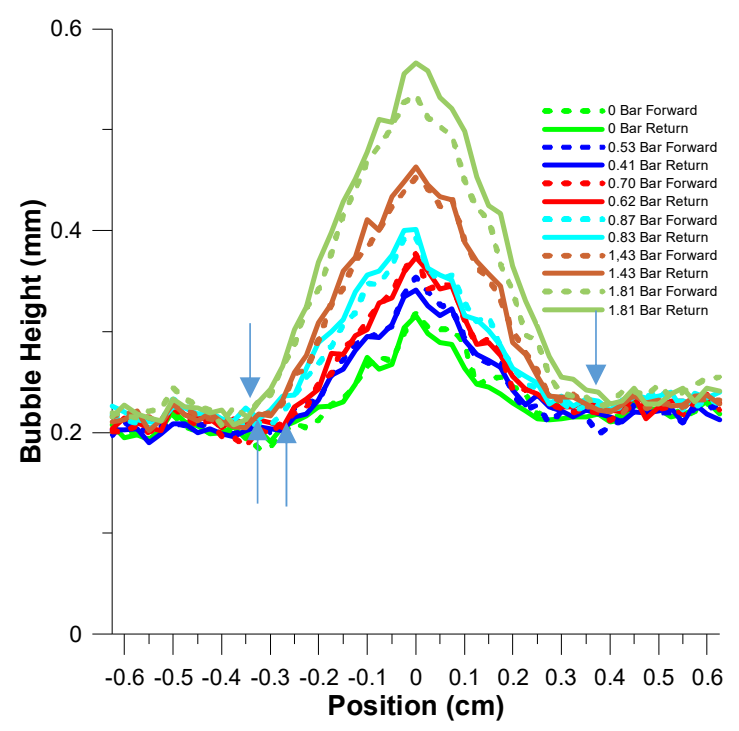

Figure 6: Blister development during one axis scan with forward (dotted line) and return movement (solid drawn line) of the laser. The arrows mark the different edge movements.

blister profile would give a better result since this would make it possible to follow the blister development. A more thorough scan of the whole blister surface in 2D would be preferable but this has a downside with respect to the increased scanning time and less ability to observe "rapid changes". The measurements here (Figure 6) are raw data without smoothing of the blister curve. Example of smoothed data are shown in Figure 8a.

\subsection{Two axis measurements}

When performing a one axis measurement only a small fraction of a potential blister development is covered. It is possible that the blister debonding happens outside the axis of measurement even though the debonding could cover a larger area. The ideal would be to instantaneous measure over the full blister area (such as a very high accuracy 3-D scanner) but our measurement a)
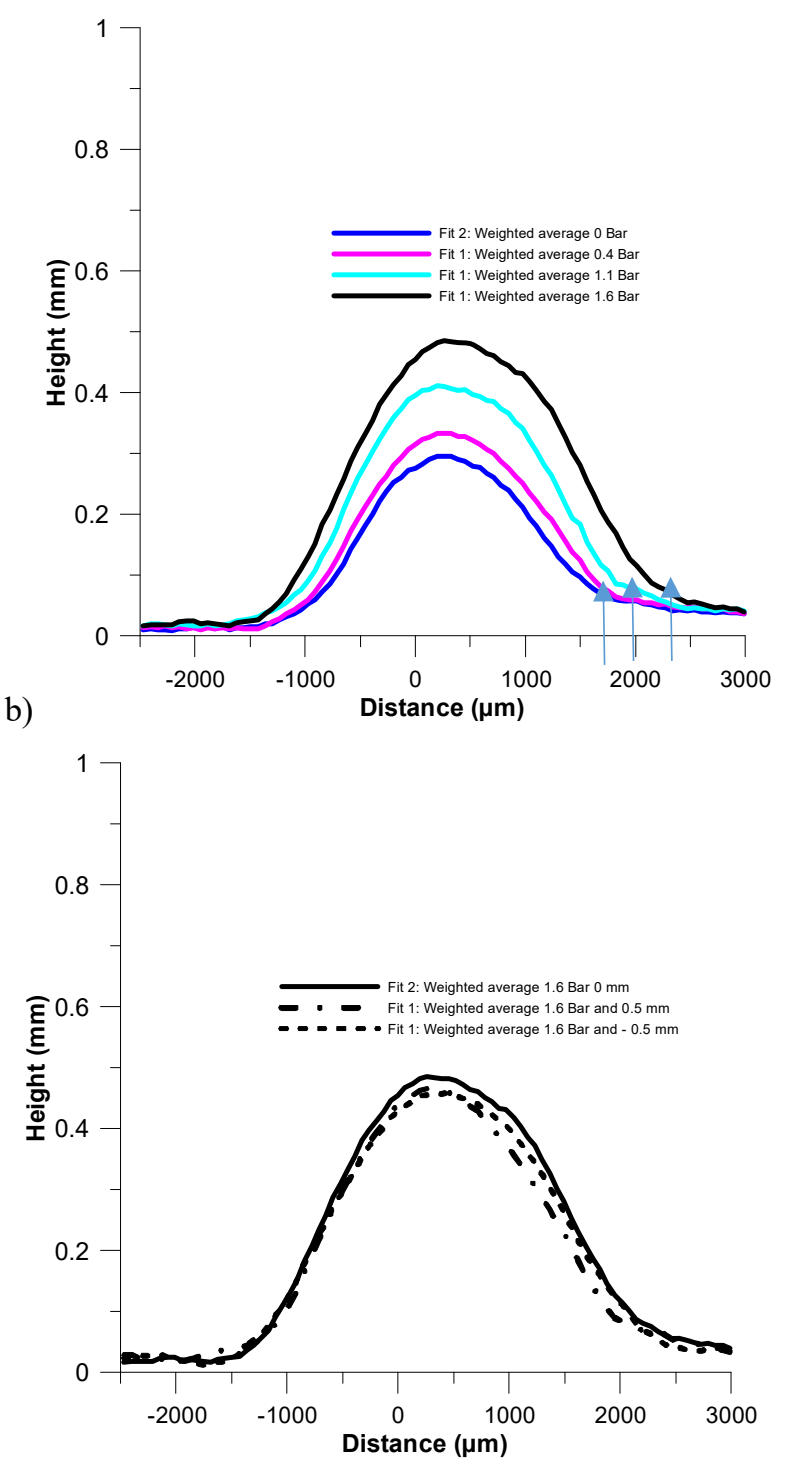

Figure 7: a) Blister development along X-axis scan (smoothed data plot for different pressures) and the arrows mark the different edge movements and b) 1.6 bar plot with $\mathrm{Y}=0, \mathrm{Y}=500 \mu \mathrm{m}$ and $\mathrm{Y}=-500 \mu \mathrm{m}$ for the plot in Figure 7a.

technique is using a laser which moves over the blister in a mapping grid with engines. A full mapping over the blister area will in our case be time consuming conflicting with the rapid development of the blister itself. We therefore have chosen to scan the blister quite accurate along the $\mathrm{X}$ axis over the blister maximum $(\mathrm{Y}=0)$ and two other positions $(\mathrm{Y}= \pm \Delta \mathrm{Y})$ giving three scanning lines over the blister. The 2-D mapping is time consuming. Another scanning along two axis would be over $\mathrm{X}=0$ and $\mathrm{Y}=0$ probably covering a larger portion of the bubble.

A 2D mapping of the blister was performed for several cases. As previous mentioned this laser technique is not ideal and even performed at engine maximum speed for the stepping engines the accuracy of the engine positioning makes it time consuming between scanning steps limited by the acceleration and retardation of the engine to maintain the position accuracy in the 
$\mathrm{X}$ and $\mathrm{Y}$ direction. All curves in Figure 7 represent the average between the forward and return scan moving the laser. The averaging of the laser input for measuring bubble height at each position to compensate for a poor laser accuracy is also a limitation. Therefor a higher laser accuracy would help but the main issue is the time scanning the blister area.

\subsection{Adhesion energy estimation from blister measurements}

The adhesion energy measurements through the blister setup on different treated flat aluminium surfaces show a large scatter for the x-linked coating. No systematic trends (Figure 8) are observed regarding the adhesion. The FEPA 1000 sandpaper treatment of the aluminium surface seem to give the highest surface energy. During the experiment we also by accident measured on the non $\mathrm{x}$-linked coating giving the lowest adhesion surface energy.

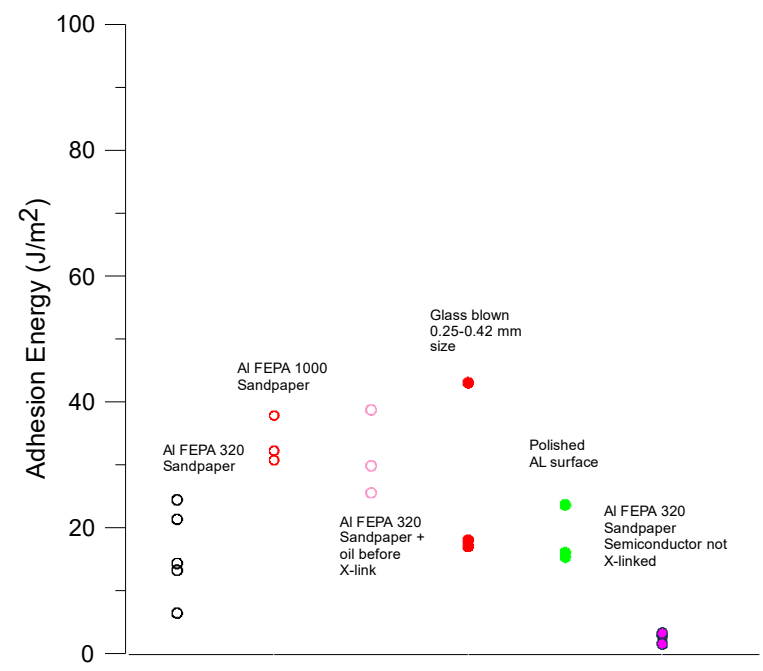

Figure 8: Summery plot of the adhesion energy measured for different treated surfaces.

\section{Conclusions}

A rig using the blister technique for measuring adhesion energy between a coating layer and a flat aluminium surface has been developed. The experimental setup is in principle able to measure the adhesion energy but the time used for scanning the blister development is an issue. Improvements regarding the scanning speed needs to be further developed.

\section{Acknowledgment}

This work was funded by Nexans Norway AS and the Research Council of Norway through the project "Aluminium in High Voltage Subsea Cables" (Project No. 245353/E20). Thanks to André Kapelrud for the help with the engine scripts.

\section{References}

[1] N. Taheri, N. Mohammadi, and N. Shahidi, "An automatic instrument for measuring of interfacial adhesion of polymeric coatings," Polymer Testing, vol. 19, pp. 959-966, 2000.

[2] J. Ordonez and C. Boehler, "A Blister-Test Apparatus for Studies on the Adhesion of Materials used for Neural Electrodes," presented at the 33rd Annual International Conference of the IEEE EMBS, Boston, Massachusetts USA, 2011.

[3] O. Altay and O. Kalenderli, "Noise reduction on partial discharge data with wavelet analysis and appropriate thresholding," in High Voltage Engineering and Application (ICHVE), 2010 International Conference on, 2010, pp. 552-555.

[4] S. White, "The Effect of Work of Adhesion on Contact of a Pressurized Blister With a Flat Surface," Master of Science, Virginia Polytechnic Institute and State University, 2001.

[5] R. J. Hohlfelder, "Bulge and Blister Testing of Thin Films and Their Interfaces," Doctor of Philosophy, Department of Materials Science and Engineering, Stanford University, 1998.

[6] L. Yongqiang, "Experimental Studies on the Mechanical Durability of Proton Exchange Membranes," Doctor of Philisophy, Virginia Polytechnic Institute and State University, 2008.

[7] M. Williams, "The continuum interpretation for fracture and adhesion," Journal of Applied Polymer Science, vol. 13, 1969

[8] K. Kristiansen, H. Faremo, and E. Ildstad, "Methods for Improving Adhesionb Between Different Materials," presented at the NORD-IS 1980 Nordic Symposium om Elektriske Isolasjonsmaterialer, Røros, 1980.

[9] H. Dannenberg, "Measurement of adhesion by a blister method," J. Appl. Polym. Sci., vol. 4, pp. 125-134, 1961.

[10] A. N. Gent and L. H. Lewansowski, "Blow-Off Pressures for Adhering Layers," Journal of Applied Polymer Science, vol. 33, pp. 1567-1577, 1987.

[11] J. A. Hinkley, "A Blister Test for Adhesion of Polymer films to SiO2," Journal of Adhesion, vol. 16, pp. 115-126, 1983.

[12] Z. Sun, K.-T. Wan, and D. Dillard, "A theoretical and numerial study of thin film delamination using the pull-off test," International Journal of Solids and Structures, vol. 41, pp. 717-730, 2004.

[13] S. Guo, K.-T. Wan, and D. Dillard, "A bending-to-stretching analysis of the blister test in the precence of tensile residual stress," International Journal of Solids and Structures, vol. 42, pp. 2771-2784, 2005. 